\title{
Optical and EUV observations of solar flare kernels
}

\author{
D. García-Alvarez ${ }^{1,2}$, C. M. Johns-Krull ${ }^{3}$, J. G. Doyle ${ }^{1}$, I. Ugarte-Urra ${ }^{1, \star}$, M. S. Madjarska ${ }^{1,4,5}$, and C. J. Butler ${ }^{1}$
}

1 Armagh Observatory, College Hill, Armagh BT61 9DG, N. Ireland

2 Smithsonian Astrophysical Observatory, 60 Garden Street, Cambridge, MA 02138, USA

e-mail: dgarcia@cfa.harvard.edu

3 Dept. of Physics and Astronomy, Rice University, Houston, TX 77005, USA

${ }^{4}$ Max-Planck-Institut für Sonnensystemforschung, Max-Planck-Str. 2, 37191 Katlenburg-Lindau, Germany

5 Dept. of Solar Physics, Royal Observatory of Belgium, Av. Circulaire 3, 1180 Bruxelles, Belgium

Received 28 June 2005 / Accepted 23 August 2005

\section{ABSTRACT}

We present high-resolution spectral observations, covering the entire optical region (3800-9000 ̊), of a solar flare observed during a multiwavelength campaign. The flare, recorded on 2002 January 11, was a medium solar flare event (GOES class C7.5). The spectral observations were carried out using the Hamilton echelle spectrograph on the coudé auxiliary telescope at Lick Observatory and with the Coronal Diagnostic Spectrometer (CDS) on board SoHO. The high signal-to-noise optical spectra are analysed using the same techniques as we applied to stellar flare data. $\mathrm{H} \alpha$ images obtained at Big Bear Solar Observatory (BBSO), plus magnetograms obtained with the Michelson Doppler Imager (MDI) on board SoHO and Transition Region And Coronal Explorer (TRACE) $1600 \AA$ were used in the flare analysis. We observe stellarlike behaviour in the main solar chromospheric activity indicators, which show either filling-in or emission during the flare. We find that the Balmer and Ca II lines show asymmetric profiles, with red-shifted wings and blue-shifted cores. This behaviour could be explained by material expanding. During the flare, the Mg I and Fe I lines show a filling-in of the line profile indicating that the flare affected the lower atmosphere. There is some evidence for pre-flare heating as seen in Fe XIX $592 \AA$. Furthermore, O v $629 \AA$ A shows an increase in flux some 10 min. before the coronal lines, perhaps indicating particle beam heating in the initial stages of the flare. We have also determined the main physical parameters at flare maximum. The electron densities and electron temperatures found for the flare imply that the Balmer emitting plasma originates in the chromosphere. The physical parameters obtained for the modelled flare are consistent with previously derived values for solar flares.

Key words. Sun: activity - Sun: photosphere - Sun: flares - Sun: chromosphere - line: formation - line: profiles

\section{Introduction}

Flares are events where a large amount of energy is released in a short interval of time, radiating at almost all frequencies in the electromagnetic spectrum. Flares (both solar and stellar) are believed to result when reconnection in the corona rapidly releases magnetic energy stored in the coronal fields (see reviews by Mirzoyan 1984; Haisch et al. 1991; Garcia Alvarez 2000). Many types of cool stars produce flares (Pettersen 1989), sometimes at levels several orders of magnitude more energetic than their solar counterparts. The exact mechanism(s) leading to the energy release and subsequent excitation of various emission features (X-ray, microwave, UV, and optical lines and continua) remains poorly understood. Solar flares are generally observed to have an impulsive phase marked by hard X-ray bursts (Dennis 1985) and microwave radio emission on time scales of seconds to minutes, indicating the presence of accelerated non-thermal particles (Lin 1974; Foukal 1990). In some cases,

^ Now in: Naval Research Laboratory, Code 7673, Washington, DC 20375, USA. optical continuum radiation is also observed (Neidig 1989). The impulsive phase is usually followed by a gradual phase, lasting minutes to hours. The impulsive phase shows continuing enhancement of chromospheric and coronal emission followed by a slow decay during the gradual phase (Svestka 1976; Tandberg-Hanssen \& Emslie 1988; Dennis \& Schwartz 1989).

The Sun, due to its proximity, allows both high spatial and spectral resolution observations of flares. Satellites such as SoHO, TRACE and recently RHESSI, are continuously monitoring high-energy radiation, and magnetograms of the surface are routinely available. Ground-based sites, such as Big Bear Solar Observatory (BBSO) and Sacramento Peak Observatory routinely monitor $\mathrm{H} \alpha$, Ca II $\mathrm{H}$ and $\mathrm{K}$, and take magnetograms. Simultaneous observations in different wavelength ranges (e.g. optical, X-rays, etc.) make it possible for the same flare to be studied at all levels in the solar atmosphere.

Despite the huge number of solar flare observations only a few experiments have studied the full optical spectrum during solar flares (Acampa et al. 1982; Donati-Falchi et al. 1984; Mauas 1990; Mauas et al. 1990). Johns-Krull et al. (1997) 
reported full wavelength optical spectra with high spectral resolution and good time resolution for a solar flare. This type of observation allows one to apply the same techniques used in the analysis of stellar flares, which provides an important link for comparing studies of solar and stellar flares. The lack of full optical range observations for solar flares is primarily due to the design of solar spectrographs which allow very high resolution line profiles but usually only small wavelength coverage. The use of cross-dispersed echelle spectra with both good spectral resolution and good time resolution has been restricted almost totally to stellar objects. Johns-Krull et al. (1997) however modified the Hamilton spectrograph at Lick Observatory to obtain solar flare observations in 1993. This allowed them to observe the spectrum of a flare at high dispersion throughout the entire optical range. Unfortunately, there was little in the way of simultaneous satellite or other ground based data taken for this flare, which limited the range of the solar atmosphere which could be studied.

In this paper we present multi-wavelength observations of a solar flare observed at 20:07 UT on 11 January 2002, although the full observing campaign included the interval between 9-15 January 2002. Three flares were observed during the campaign, in this paper only one solar flare will be reported, a GOES C7.5 event, which had the most simultaneous coverage. The objective of this campaign was to monitor for flare events with the same sort of spectral coverage as is normally obtained in stellar events. Observing time was allocated for this project at Lick Observatory, Big Bear Solar Observatory, the CDS and MDI instruments onboard the SoHO, plus TRACE. Observations and data analysis are described in Sect. 2; while in Sect. 3 we discuss the flare evolution as seen by the different instruments. In Sect. 4 we analyse the behaviour of the chromospheric and photospheric lines during the flare, and the chromospheric and coronal flare energy budget. In Sect. 5 we analyse the main chromospheric physical parameters of the emitting region during the flare with the conclusions given in Sect. 6 .

\section{Instrumentation and observational material}

\subsection{Lick observations}

The Hamilton echelle spectrograph (Vogt 1987) and the telescopes that feed the spectrograph, were not designed for solar observations. As a consequence of this, some unusual steps were needed to observe solar flares. We have followed the same observational setup, with some minor modifications, used by Johns-Krull et al. (1997) in order to obtain the present data. The 0.6 m Coude Auxiliary Telescope (CAT) was used to feed the spectrograph. The primary mirror was covered with a mask that stepped the aperture down to a circle of approximately $20 \mathrm{~cm}$ diameter. The CAT itself is fixed to the wall of the Observatory and is fed by a siderostat. This system feeds a white-light image to the spectrograph slit, which, for this study was $5.5^{\prime \prime}$ wide by $10.0^{\prime \prime}$ long. In order to cut down on the flux delivered to the spectrograph CCD, we inserted a $B K 38$ filter behind the spectrometer slit. To further cut down on the flux, a piece of mylar was inserted in the filter holder along with the $B K 38$ glass. Optical wavelength coverage was from 3800 to $9000 \AA$. This combination provides a spectral resolution of $R \sim 45000$ and a temporal resolution of $\sim 2 \mathrm{~min}$. To improve the contrast of active regions on the Sun (compared to the image delivered to the spectrograph slit) and to enable the identification of solar flares, a $0.6 \AA$ Daystar $\mathrm{H} \alpha$ filter was placed in front of the slit viewing guide camera. The siderostat could then be used to point the CAT to any feature visible in the $\mathrm{H} \alpha$ image, in our case, flare kernels.

The spectra were reduced with customized IDL software using a standard procedure discussed by Valenti (1994). This includes tracing each spectral order on the chip, flat-fielding and subtracting scattered light measured between orders. Use of the combined mylar and $B K 38$ filter behind the slit resulted in excess fringing which is not fully removed in the standard flat-fielding procedure. In order to better remove this, we divide an extracted spectrum of a quiet region taken with the filter mounted behind the slit by an extracted spectrum of a quiet region taken without this filter in place. This results in a spectrum of the excess fringing which was then divided into each exposure taken with the combined mylar and $B K 38$ to remove the fringes. Visual inspection indicates that residual fringing is less than $2 \%$. In addition, the results shown below using this data are differential in nature (flare minus quiet, both observed with the combined mylar and $B K 38$ filter), making this fringing less of a concern. Finally, wavelength calibration was accomplished by fitting a two-dimensional polynomial to $n \lambda$ (where $n$ is the echelle order number) as a function of thorium-line location in a lamp spectrum.

At BBSO, the active region NOAA 9773, where the flare occurred, was observed throughout the campaign. Figure 1 shows $\mathrm{H} \alpha$ images of the active region, taken at BBSO from the flare onset until the end of the gradual phase. Several eruptive centers can be observed during the flare onset. The flaring kernel site observed at Lick Observatory is marked in each image with the lower left of the 2 rectangles

\subsection{TRACE observations}

The TRACE (Handy et al. 1999) observations used in this study were obtained from 20:02 UT until 20:32 UT on 2002 January 11 . The observations were taken in the $1600 \AA$ passband which contains the $\mathrm{C}$ I lines, Fe II lines and UV continuum with a significant contribution from both the C IV 1548 and $1550 \AA$ lines. The passband width is $275 \AA$. The exposure time of the images varies from 0.15 to $0.43 \mathrm{~s}$. The TRACE angular pixel size is $0.5^{\prime \prime}$ which gives a $1^{\prime \prime}$ spatial resolution. The data were flat field corrected, normalized for the exposure and pointing corrected for the corresponding channel. The images (Fig. 2) are used to study the UV emission which during the flare is probably dominated by the C IV doublet emission. The $\mathrm{C}$ IV formation temperature is $100000 \mathrm{~K}$.

\subsection{MDI observations}

The MDI (Scherrer et al. 1995) observations were obtained at 20:02 and 20:07 UT in a full-disk mode. The resulting images have $34^{\prime} \times 34^{\prime}$ field-of-view (FOV). The angular pixel size 

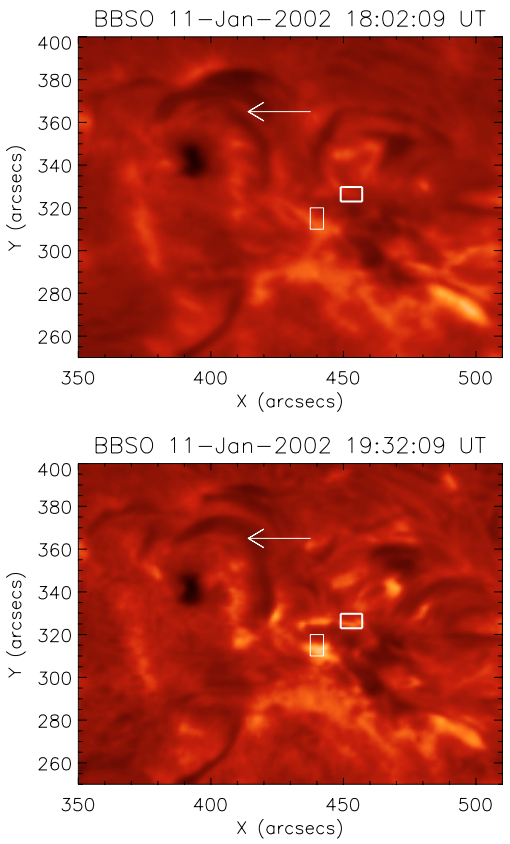
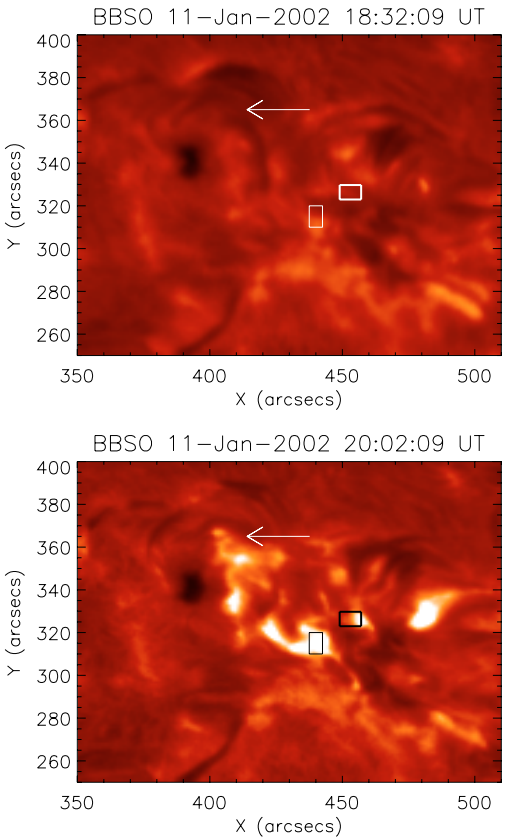
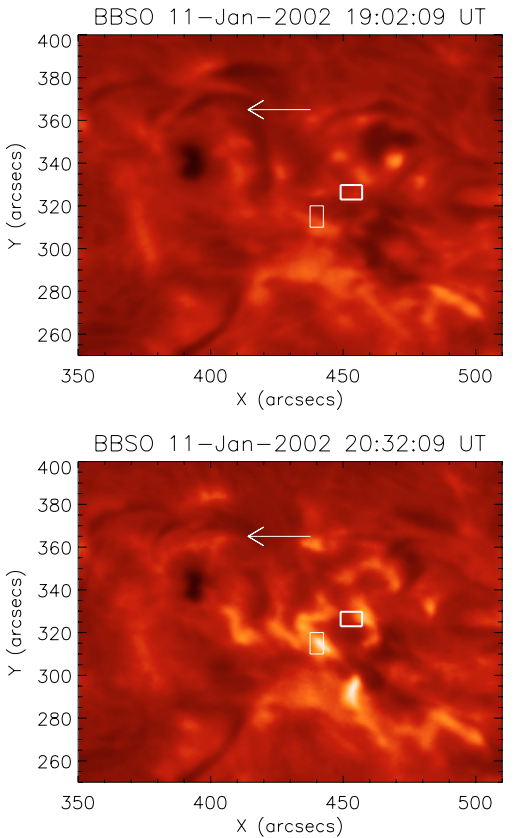

Fig. 1. BBSO images of the active region NOAA 9773 that produced the 2002 January 11 solar flare. They show the evolution in time of the flare in the $\mathrm{H} \alpha$ line. In each image the position of the flaring knot is marked with a rectangle that represents the size of the optical slit. The $\mathrm{CDS}$ area of integration is also indicated (thicker line rectangle). The arrow shows the position of a disappearing filament.
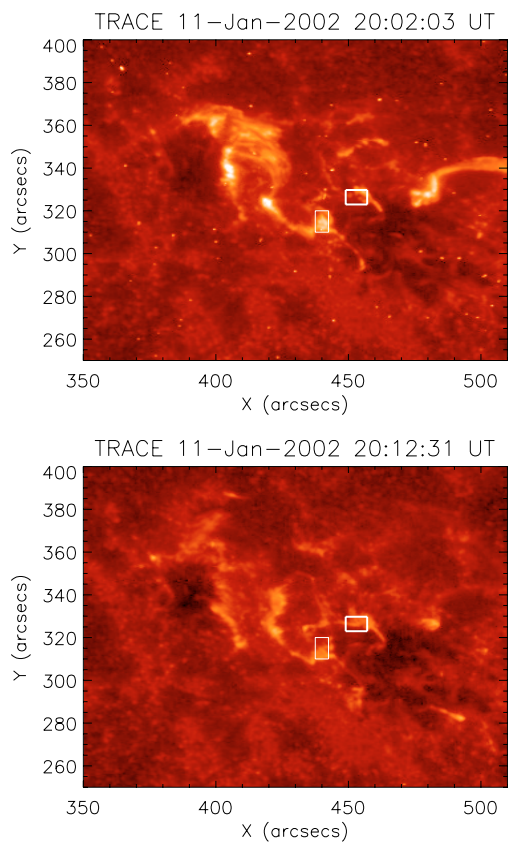
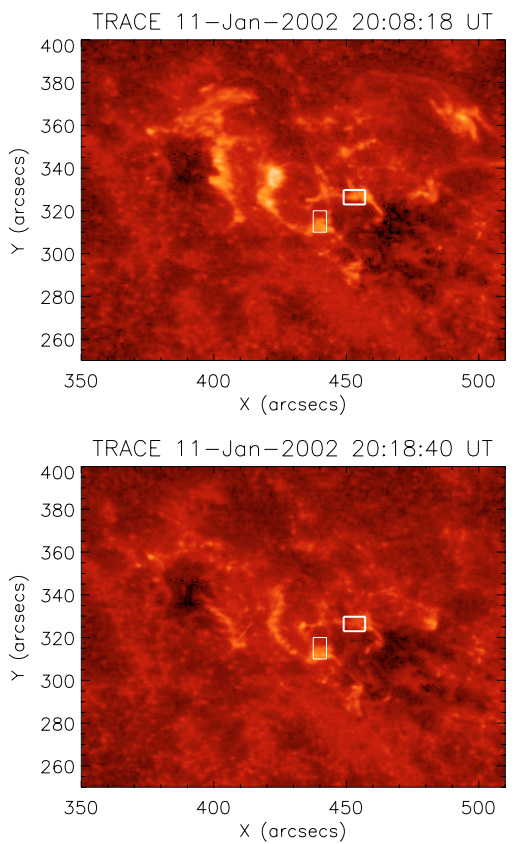
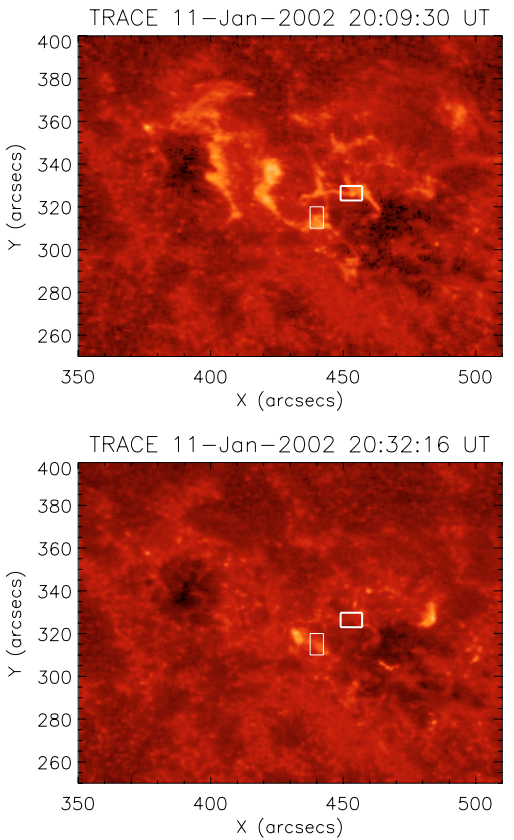

Fig. 2. The evolution of the 2002 January 11 solar flare as seen in the TRACE $1600 \AA$ A images. In each image, the position of the Lick slit is shown with a thin line rectangle. The CDS area of integration is also indicated (thick line rectangle).

is $1.98^{\prime \prime}$ pixel $^{-1}$. We used Level-1.5 calibrated magnetograms for this study. The magnetograms were additionally corrected for a flux saturation for magnetic flux above $1200 \mathrm{G}$ (for more details see Green et al. 2003, and the citation therein). The images were rotated to the time 20:32 UT (as all the other SoHO and TRACE images used in this paper). They were also converted in order to correspond to the TRACE view point (Earth view).

\subsection{CDS observations}

The Coronal Diagnostic Spectrometer (CDS) (Harrison et al. 1995) onboard SoHO provided EUV spectral coverage of one of the flare kernels of the flare (see Figs. 1 and 2). Rasters of $20^{\prime \prime} \times 202^{\prime \prime}$ made out of five adjacent $6 \mathrm{~s}$ exposures, using the $4^{\prime \prime} \times 240^{\prime \prime}$ slit and pixel scale of 3.36" in the NorthSouth direction, were obtained for four spectral windows 

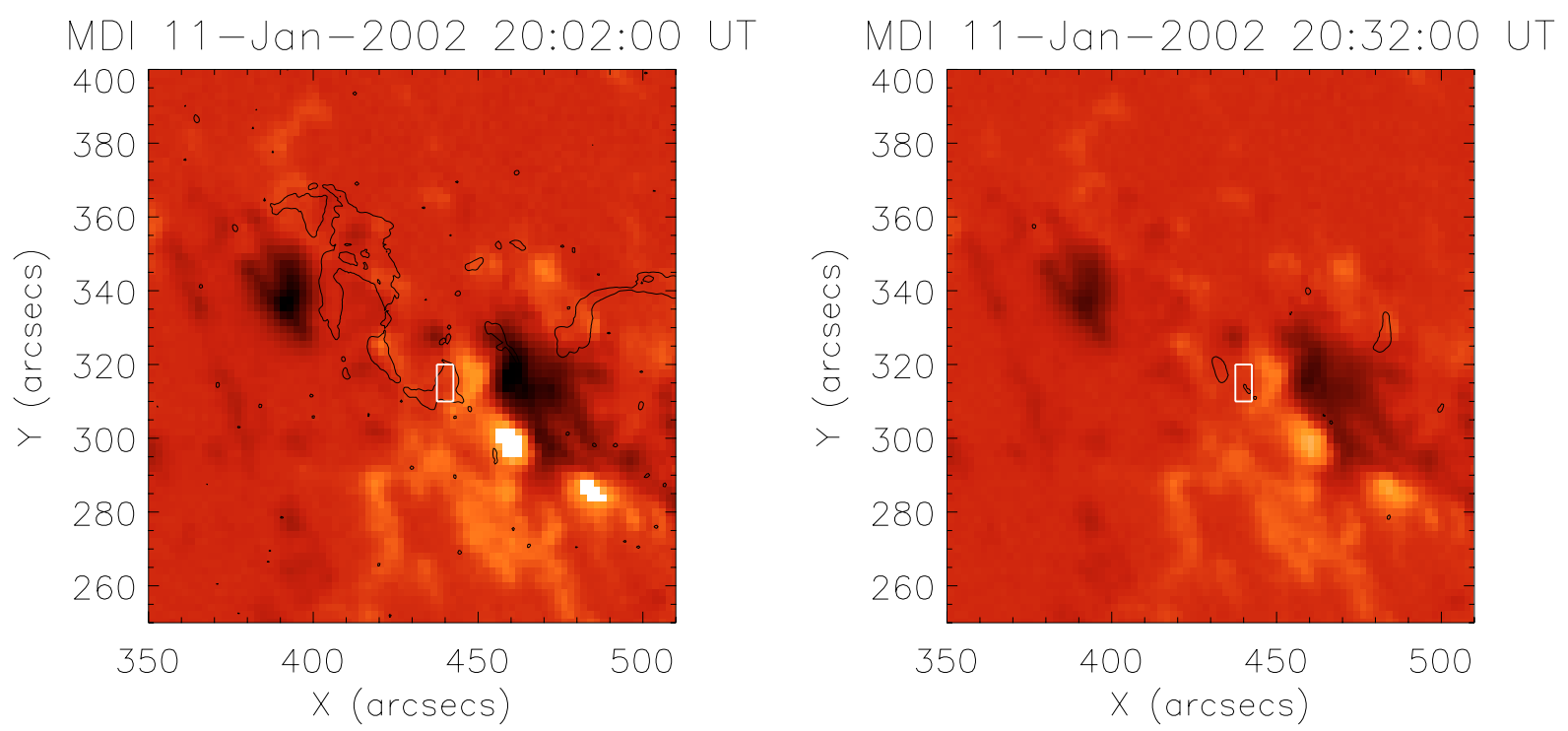

Fig. 3. MDI magnetograms (scaled from -2000 to $2000 \mathrm{G}$ ) with overplotted the TRACE contour map for the corresponding time and the position of the Lick observatory slit.

centered at Fe XIX $592.2 \AA$, Fe XVI $360.8 \AA$, Mg X 624.9 and O v $629.7 \AA$. The time needed to build every raster was $84 \mathrm{~s}$ which resulted in an average cadence of $106 \mathrm{~s}$ between the datapoints. The position of the CDS slit over a kernel is noted in Figs. 1 and 2. CDS pointing is always given in advance to the observations while the Lick pointing was done during the flare onset.

Standard reduction was applied to the CDS data correcting for bias, flat-field, cosmic rays, and instrumental effects such as horizontal shifts due to the rotation of the scan mirror and rotation and tilt in the spectrum due to the misalignment between grating and detector, and grating and slit. Standard software was used in the data reduction and line fitting. An area of 8.1" $\times 6.7^{\prime \prime}$ was tracked within the rasters from 19:43 UT to 21:00 UT and its emission integrated producing a lightcurve for each of the cited spectral lines (see Fig. 4 later).

\subsection{Instrumental offset corrections}

The pointing offset of the instruments used in the present study was corrected using the TRACE images as a reference. First, all the images used in this study were rotated to the time 20:32 UT. Then the BBSO H $\alpha$ and TRACE $1600 \AA$ images were coaligned using the sunspots as a reference point. A $20^{\prime \prime}$ Solar_Y offset was found. The next step was to coalign TRACE and SOHO instruments, using MDI images. Both instruments recorded white light images which were used to determine the offset via cross-correlation. That offset was also used to compare TRACE and CDS. Finally, the entire TRACE dataset was visually inspected and compared for reassurance.

\subsection{GOES}

In addition, we used the GOES (1-8 $\mathrm{A})$ and $(0.5-4 \AA)$ passband data in order to compare the coronal and chromospheric behaviour during the flare and obtain a rough estimate of the energy released in this region.

\section{The flare evolution}

On 2002 January 11 we observed the flare event discussed here. The flare onset was first detected by the BBSO instrument at around 19:55 UT. The first optical spectrum recorded at Lick Observatory was at flare maximum at 20:07 UT. The flare took place in active region NOAA 9773 and was classified as C7.5. This implies, based on the soft X-ray (SXR) emission monitored by the GOES satellite, an emitted flux of $7.5 \times 10^{-6} \mathrm{~W} \mathrm{~m}^{-2}$ at the Earth or $5.3 \times 10^{24} \mathrm{erg} \mathrm{s}^{-1}$ at the solar surface. This was therefore a medium size flare.

Active region NOAA 9773, N14W27, was classified following the Mt Wilson magnetic classification introduced by Hale et al. (1919) as a $\beta \gamma \delta / \beta \gamma \delta$, which implies a bipolar group with an irregular distribution of polarities. The magnetic flux density in the active region ranged from -2000 to $3000 \mathrm{G}$ at 19:02, 19:32 and 20:02 UT while the magnetogram at 20:32 UT gives values from -1480 to $1230 \mathrm{G}$ (see Fig. 3). The total unsigned magnetic flux derived from the measured MDI longitudinal magnetic field decreased by about $30 \%$ from 20:02 to 20:32 UT. Note that this is the flux measured only in the FOV shown in Fig. 3.

The $\mathrm{H} \alpha$ images consist of several brights kernel during the impulsive phase and bright post-flare loops during the gradual phase. A filament was present several arcsecs north of the flare eruption from at least 18:02 UT and was still present at the start of the flare at 20:02 UT. By 20:32 UT it had disappeared. Unfortunately, the time resolution does not permit us to comment on its relation to the observed flare activity. The higher time resolution data are the TRACE observations.

All the optical spectral observations reported in this work were made on the marked kernel (see Fig. 2). The quiescent solar spectra were taken near the active region, but almost one 


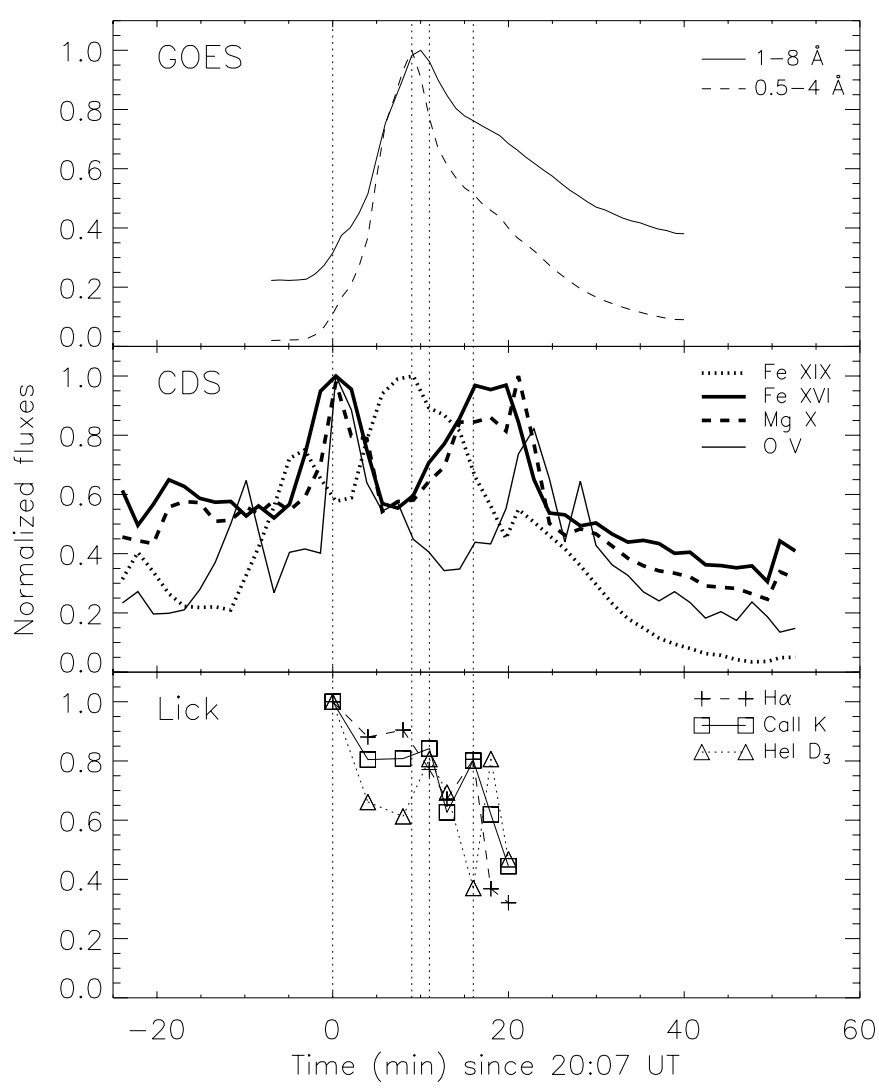

Fig. 4. Evolution of the flare as seen by GOES (top panel), CDS: Fe XIX $592 \AA$, Fe XVI $360 \AA$, Mg X $624 \AA$ and O V $629 \AA$ (middle panel) and Lick: $\mathrm{H} \alpha$, Ca II $\mathrm{K}$ and $\mathrm{He} \mathrm{I} \mathrm{D}_{3}$ lines (bottom panel).

day after the flare. This allows us to minimize the presence of small-scale structures other than the flare itself. However, a shorter interval between the flare and quiet spectra would have been desirable.

The lower panel in Fig. 4 shows the evolution of the $\mathrm{H} \alpha, \mathrm{He} \mathrm{I} \mathrm{D}_{3}$ and $\mathrm{Ca}$ II $\mathrm{K}$ lines. All three lines have a similar behaviour, although $\mathrm{He} \mathrm{I}$ is more impulsive-like. The photospheric lines (MgI Triplet) and metallic lines (Fe I) have their maximum enhancement roughly at the same time as the other chromospheric lines (see Table 1). The decline in the $\mathrm{Ca}$ II $\mathrm{K}$ line is delayed with respect to the Balmer lines.

As the CDS lines were taken slightly off-set from the optical flare kernel, direct comparison with the optical data is difficult. O v 629 produces a peak at around 19:57 UT, some $10 \mathrm{~min}$ before the coronal lines peak. Such behaviour has been seen many times from SMM data, where the transition region lines are seen to correlate to within $1 \mathrm{~s}$ of hard X-ray bursts of at least $50 \mathrm{keV}$ (Cheng et al. 1981; Woodgate et al. 1983). CDS has also observed before such a precursor brightening with no coronal counterpart (Brosius 2003). It has been interpreted in terms of particle beams which deposit much more energy in the chromosphere and transition region than in the corona (see Brosius 2003; Brosius \& Phillips 2004, for a further discussion), which would explain the absence of a coronal response. $\mathrm{O} \mathrm{V}$ produces another two peaks, one at 20:07 UT and a further one at 20:29 UT. Mg X and Fe XVI also peak at 20:07 UT with a further increase in flux starting at 20:20 UT, giving a broad peak at 20:25 UT. The Fe XVI initially starts to increase in flux at 20:02 UT. The hottest line observed, Fe XIX shows a very different behaviour; first showing a peak at 20:02 UT and a second larger increase at 20:15 UT. That peak is simultaneous to the one observed in GOES data (20:16 UT) which starts rising at 20:04 UT. It is unclear whether the first peak seen in the Fe XIX line corresponds to the pre-flare heating as noted by other authors, e.g. Harra et al. (2001). The problem here is that CDS is observing the activity at a single flare kernel, while GOES observes the total flare integrated flux increase. A blend with a cool line does not seem to be the reason, because the precursor brightening does not show up in the Fe XIX lightcurve. The behaviour after the GOES and Fe XIX peak at around 20:16 UT can be better reconciled if we think about hot loops seen in Fe XIX which slowly cool down passing through different temperature regimes and being observed first in Fe XVI and $\mathrm{Mg} \mathrm{X}$, and later in $\mathrm{OV}$, spanning around $20 \mathrm{~min}$. The sharp increase in the $\mathrm{MgX}$ lightcurve $20 \mathrm{~min}$ after the flare maximum (20:07 UT) is an artifact consequence of the solar rotation tracking and the dimensions of the CDS pixels. It shows up also in Fe XIX. That is not the case in the rest of the instances where solar rotation is corrected.

\section{Chromospheric activity indicators}

The wavelength range covered by our observations (3800-9000 $)$, allows us to probe the behaviour of different chromospheric lines during the solar flare, and compare them with their stellar counterparts. Our solar observations are relative-intensity, so we have to transform them into fluxes. In order to make this transformation, we have first corrected for limb darkening (Pierce \& Waddell 1961) at the position of the flare $\left(20^{\circ}\right.$ from disk center) and then calibrated our observed continuum fluxes by reference to standard solar continuum fluxes from Allen (1976). We combined those values with the excess equivalent width $(E W)$ of the Balmer lines shown in Table 1.

In order to obtain the excess $E W$, we first subtract the quiescent solar spectrum from the flare spectra. This is the normal procedure followed in stellar flare observations where many of the lines are in emission even when quiescent. Then, we measure the $E W$ with respect to a local continuum interpolated from a linear fit to line-free nearby continuum portions included within the spectral interval. In this analysis we consider positive and negative values of the $E W$ as referring to lines in absorption and emission, respectively. Table 1 also shows the excess $E W$ for other chromospheric activity indicators such as

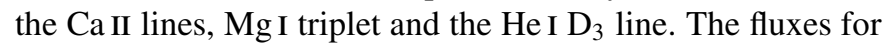
the Balmer lines $(\mathrm{H} \alpha$ to $\mathrm{H} \epsilon)$ and $\mathrm{Ca}$ II are reported in Table 2.

\subsection{The Balmer lines}

Figure 5 shows the flare plus quiet Sun line profile, the quiet Sun profile, and the subtracted profile for the main chromospheric activity indicators observed during flare maximum, at 20:07 UT. We note that all the Balmer lines show a filling-in during flare maximum, which decreases as the flare evolves (see Table 1). We note that the peak intensity in $\mathrm{H} \alpha$ is greater 
Table 1. Flare observations and excess equivalent widths in angstroms for the activity chromospheric indicators observed during the solar flare at 20:07 UT on 11 January 2002. The sign of the $E W$ follows the usual convention (negative values for emission lines).

\begin{tabular}{ccccccc}
\hline \hline Frame & $\mathrm{UT}$ & $\mathrm{H} \alpha$ & $\mathrm{H} \beta$ & $\mathrm{H} \gamma$ & $\mathrm{H} \delta$ & $\mathrm{H} \epsilon$ \\
\hline 1 & $20 \mathrm{~h} 07$ & -738 & -281 & -180 & -159 & -134 \\
2 & $20 \mathrm{~h} 11$ & -650 & -254 & -167 & -130 & -112 \\
3 & $20 \mathrm{~h} 15$ & -667 & -209 & -165 & -133 & -108 \\
4 & $20 \mathrm{~h} 18$ & -569 & -227 & -157 & -140 & -113 \\
5 & $20 \mathrm{~h} 20$ & -495 & -176 & -137 & -105 & -94 \\
6 & $20 \mathrm{~h} 23$ & -594 & -255 & -133 & -94 & -95 \\
7 & $20 \mathrm{~h} 25$ & -272 & -99 & -90 & -52 & -64 \\
8 & $20 \mathrm{~h} 27$ & -237 & -91 & -65 & -27 & -54 \\
\hline
\end{tabular}

\begin{tabular}{cccccccccc}
\hline \hline Frame & Ca II K & Ca II H & Ca II & Ca II & Ca II & He I & Mg I & Mg I & Mg I \\
& $3933 \AA$ & $3968 \AA$ & $8498 \AA$ & $8542 \AA$ & $8662 \AA$ & $5875 \AA$ & $5167 \AA$ & $5172 \AA$ & $5183 \AA$ \\
\hline 1 & -500 & -589 & -591 & -456 & -439 & 62 & -79 & -75 & -98 \\
2 & -403 & -473 & -564 & -423 & -414 & 41 & -113 & -86 & -103 \\
3 & -404 & -499 & -500 & -393 & -377 & 38 & -91 & -67 & -103 \\
4 & -421 & -521 & -472 & -352 & -343 & 50 & -66 & -51 & -93 \\
5 & -408 & -500 & -421 & -303 & -292 & 43 & -49 & -35 & -61 \\
6 & -401 & -492 & -494 & -346 & -347 & 23 & - & - & - \\
7 & -310 & -359 & -341 & -227 & -208 & 50 & - & - & - \\
8 & -222 & -265 & -285 & -185 & -186 & 29 & - & - & - \\
\hline
\end{tabular}

Table 2. Fluxes in units of $10^{6} \mathrm{erg} \mathrm{s}^{-1} \mathrm{~cm}^{-2}$ at the solar surface for the Balmer, Ca II and He I lines observed during the solar flare at 20:07 UT on 11 January 2002.

\begin{tabular}{cccccccccccc}
\hline \hline Frame & $\mathrm{H} \alpha$ & $\mathrm{H} \beta$ & $\mathrm{H} \gamma$ & $\mathrm{H} \delta$ & $\mathrm{H} \epsilon$ & $\mathrm{Ca}$ II K & $\mathrm{Ca}$ II H & Ca II & Ca II & Ca II & He I \\
$8933 \AA$ & $3968 \AA$ & $8498 \AA$ & $8542 \AA$ & $8662 \AA$ & $5875 \AA$ \\
\hline 1 & 6.38 & 3.25 & 2.19 & 1.88 & 1.52 & 2.97 & 3.97 & 3.34 & 2.56 & 2.41 & 0.61 \\
2 & 5.62 & 2.94 & 2.03 & 1.53 & 1.27 & 2.39 & 3.19 & 3.19 & 2.37 & 2.27 & 0.41 \\
3 & 5.77 & 2.42 & 2.01 & 1.57 & 1.23 & 2.40 & 3.36 & 2.83 & 2.20 & 2.07 & 0.38 \\
4 & 4.92 & 2.62 & 1.91 & 1.65 & 1.28 & 2.50 & 3.51 & 2.67 & 1.97 & 1.88 & 0.49 \\
5 & 4.28 & 2.03 & 1.67 & 1.24 & 1.07 & 1.86 & 3.37 & 2.38 & 1.70 & 1.60 & 0.43 \\
6 & 5.14 & 2.95 & 1.62 & 1.11 & 1.08 & 2.38 & 3.31 & 2.79 & 1.94 & 1.90 & 0.23 \\
7 & 2.35 & 1.14 & 1.10 & 0.61 & 0.73 & 1.84 & 2.41 & 1.93 & 1.27 & 1.14 & 0.50 \\
8 & 2.05 & 1.05 & 0.79 & 0.32 & 0.61 & 1.32 & 1.78 & 1.61 & 1.04 & 1.02 & 0.29 \\
\hline
\end{tabular}

than in $\mathrm{H} \beta$ and $\mathrm{H} \gamma$. In the stellar case, the strongest Balmer line is usually $\mathrm{H} \gamma$ or $\mathrm{H} \delta$ as reported for a flare on AD Leo (García-Alvarez et al. 2003). In solar flares, $\mathrm{H} \beta$ and $\mathrm{H} \gamma$ are sometimes observed with a larger flux than $\mathrm{H} \alpha$ as reported by Zirin et al. (1982).

\subsection{The $\mathrm{Ca} \| \mathrm{H}$ and $\mathrm{K}$ lines and the Ca II infra-red triplet}

Enhanced emission cores in the Ca II H and $\mathrm{K}$ lines are the primary optical indicators of chromospheric activity. These lines are sensitive to changes in the electron density and temperature in the chromosphere. Figure 5 shows the $\mathrm{Ca}$ II $\mathrm{H}$ and $\mathrm{K}$ lines turning into emission during the flare. We also note that the $\mathrm{Ca}$ II $\mathrm{H}$ and $\mathrm{K}$ lines show central reversal. This is a common feature in solar plage and network features (Bocchialini \& Gouttebroze 1996) and in stellar emission lines on active red dwarfs.

The second feature we observe in the Ca II $\mathrm{H}$ and $\mathrm{K}$ lines, is that the line shape is not symmetrical, with two peaks of different intensity, as if the reversal were not at the center of the line. This may be the signature of changes in the nature of the original source and/or in the state of the atmosphere. It may suggest that the rest frame of the line differs between the source region and the overlying atmosphere, with relative motions of a few $\mathrm{km} \mathrm{s}^{-1}$ along the line of sight. Apart from these features, we also note that, during the flare, the excess 

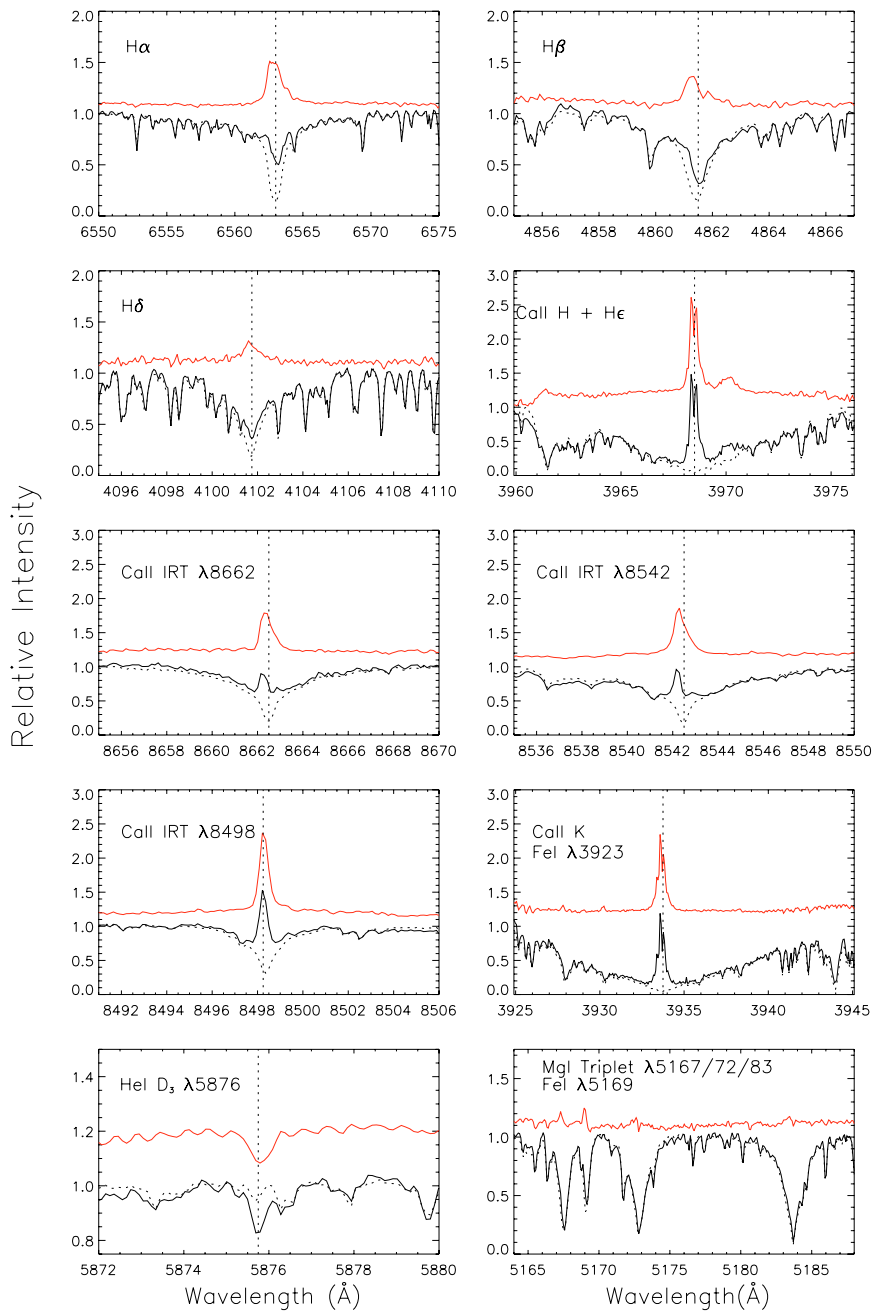

Fig. 5. The line profiles for the Balmer lines, the Ca II lines, the $\mathrm{He} \mathrm{I}_{3}$ line, the Mg I triplet and a few Fe I lines, at flare maximum, 20:07 UT. The lower solid line shows the observed flare plus quiet Sun for all the profiles, while the dotted line shows the quiet-Sun line profile. The upper solid line is the flare minus quiet-Sun, offset by 1.1 for better display. The dotted vertical lines correspond to the rest wavelength.

emission in the Ca II $\mathrm{H}$ line is, on average, around 20\% larger that the Ca II $\mathrm{K}$ line (see Table 1).

The Ca II infra-red triplet (IRT) $18498, \lambda 8542$, and $\lambda 8662$ lines are another important stellar chromospheric activity indicator (Linsky et al. 1979; Dempsey et al. 1993; Gunn $\&$ Doyle 1997). These lines are formed in the lower chromosphere of the Sun (Vernazza et al. 1981). Foing et al. (1989) found that the Ca II IRT lines correlate well with the Ca II $\mathrm{H}$ and $\mathrm{K}$ peaks. Unlike the bluer Ca II lines, the Ca II IRT lies in a region with a well-defined continuum which makes the analysis simpler. Shine \& Linsky $(1972,1974)$ reported that in the quiet solar atmosphere these lines are in absorption, while they show core brightening and eventually develop selfreversed emission when observed in bright plages. These authors concluded that the emission resulted from a steepening of the temperature gradient in the chromosphere. Figure 5 shows the CaII IRT lines turning into emission during the flare. They keep this state well into the gradual phase. The Ca II $\lambda 8498$ line is the strongest of all the IRT lines (see
Table 1). It shows an excess emission around $39 \%$ and $43 \%$ larger than the Ca II $\lambda 8542$ and $\lambda 8662$ lines respectively. The excess emission of the Ca II $\lambda 8498$ line is roughly the same as for $\mathrm{Ca}$ II $\mathrm{H}$.

The decline in all the Ca II lines is delayed with respect to the Balmer lines. The rapid increase in the continuum and Balmer line fluxes observed during flares (García-Alvarez et al. 2002; Houdebine 2003) is consistent with an increase of the local electron density due to increased photo-ionization of Hydrogen (Houdebine et al. 1991). However, increases in the Ca II photo-ionization continua would deplete the Ca II ground state population. The increase in electron density creates larger collisional excitation rates that could balance the previous effect. These two effects would force a different behaviour of the Ca II lines compare to the Balmer lines as we obtained from the observations.

\subsection{The $\mathrm{HeCa}$ । $\mathrm{D}_{3}$ line}

All the changes observed in the spectral lines during the flare produce either filling-in or outright emission. $\mathrm{He}_{\mathrm{I}} \mathrm{D}_{3}$ has been previously detected in absorption in active stars where it was attributed to plages or coronal radiation (Huenemoerder 1986). It has also been seen in emission during stellar flares (Montes et al. 1997, 1999; Oliveira \& Foing 1999). Landman (1981) studied high resolution spectra of the $\mathrm{He}$ I $\mathrm{D}_{3}$ line in solar plage regions. In the Sun, it is in emission in the strongest flares (Tandberg-Hanssen 1967) and appears in absorption in weak to medium flares and in eruptive prominences (Zirin 1988). Zirin (1975) reported that the mechanism for the appearance of the $\mathrm{He} \mathrm{I}_{3}$ line in absorption involves ionization by coro-

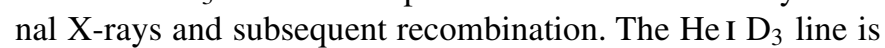
harder to excite than the Balmer lines, due to its higher excitation potential. Andretta \& Giampapa (1995) suggested, on the basis of their NLTE calculations that some of the He I spectral features in the Sun and late-type stars could be linked to regions of enhanced UV and X-ray emission.

Johns-Krull et al. (1997) found a very distinct behaviour for this line, however, their analysis was for a much larger solar flare. Andretta \& Giampapa (1995) found that this line can be formed in two different atmospheric regions: in the upper chromosphere at temperatures of $7000-8000 \mathrm{~K}$ and in a plateau above $20000 \mathrm{~K}$. They conclude that in weaker chromospheres, formation in the upper chromosphere dominates.

In the present flare, we observe $\mathrm{He} \mathrm{I}_{3}$ in absorption during the flare, while it is undetectable in the quiescent state. Variability in its $E W$ is clearly observed during the flare in overall agreement with the variability observed in other lines, although it is more impulsive-like.

\subsection{The photospheric lines}

Strong neutral metal absorption lines that are formed in the lower chromosphere/temperature minimum region are very interesting for solar-stellar activity studies. The Mg I triplet consists of low excitation lines emitted when the low chromosphere is heated, usually at foot-points of flux loops passing 


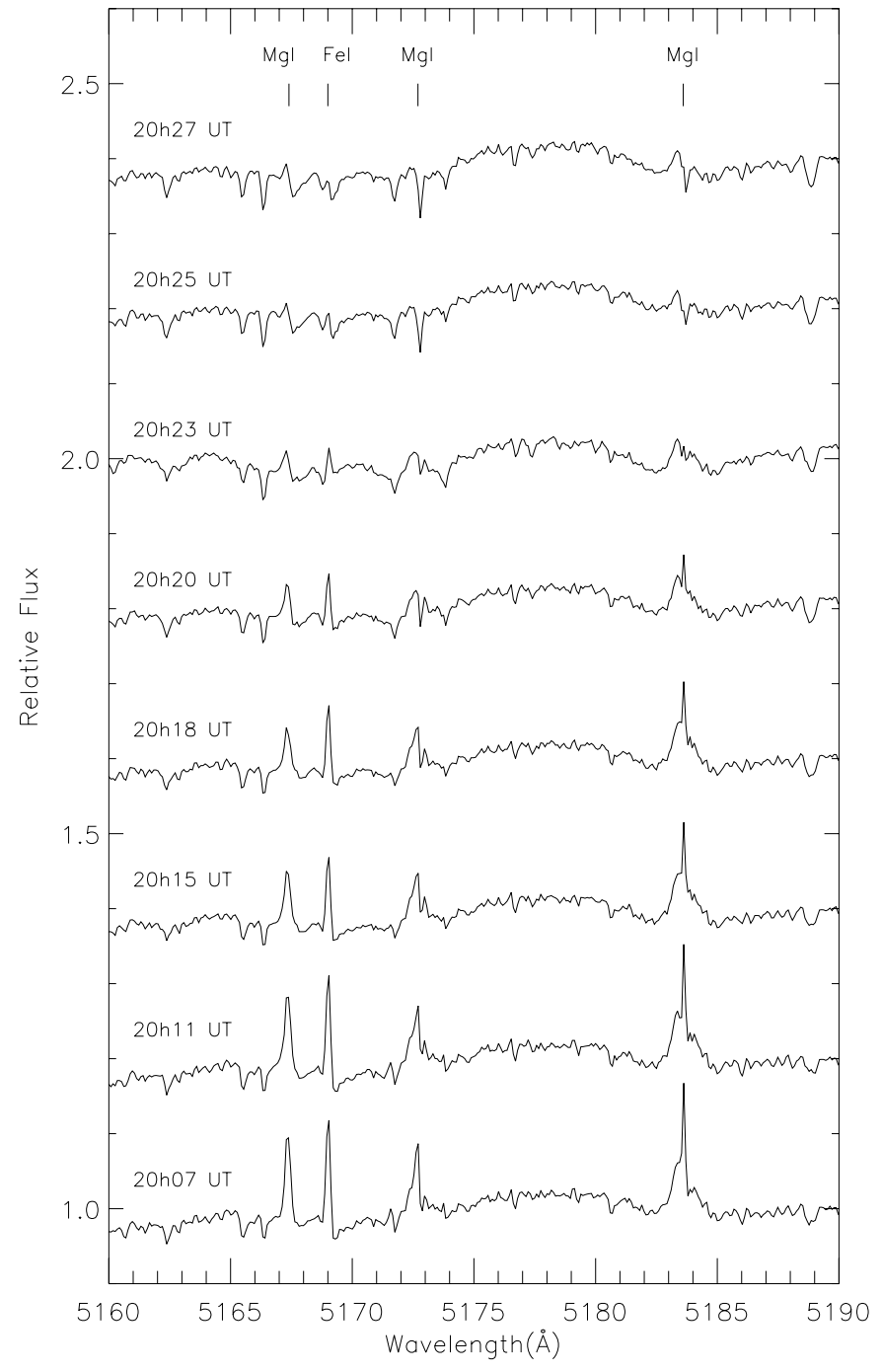

Fig. 6. Time series of the flare minus quiet Sun spectra showing Mg I and $\mathrm{Fe}$ I lines during the 2002 January 11 solar flare.

through the flare. It is a good diagnostic of photospheric activity (Basri et al. 1989). A filling-in of these lines has been found in some RS CVn systems (Gunn \& Doyle 1997; Montes \& Ramsey 1998). This is observed in the last panel of Fig. 5. Together with the filling-in of Fe I lines, which are formed in the upper photosphere. This suggests that the flare affected the lower chromosphere. We found in the quiet Sun spectra no excess equivalent width for $\mathrm{Mg}$ I or Fe I. Figure 6 shows a time series of the analysed photospheric lines during the flare. Only a small filling-in was found for the Na I doublet. This is surprising since both neutral metals, $\mathrm{Mg}$ I and $\mathrm{Na}$ I, form in the lower chromosphere and, taking into account that the ionization energies are 7.6 and $5.1 \mathrm{eV}$ respectively, one would expect the Na I Doublet to show a stronger filling-in than the Mg I Triplet. A similar behaviour was found by Gunn \& Doyle (1997) in the RS CVn system ER Vul.

\subsection{Line asymmetries}

It is well known that the profiles of some strong chromospheric emission lines in the spectra of solar flares have asymmetries (see e.g. Svestka 1976, and references therein). It is important to measure these asymmetries during flares as they may allow us to measure the velocities of the emitting plasma. Blue and red asymmetry can be found in the same flare, depending on the phase. High temporal resolution is required in order to perform this kind of study. Several authors (i.e. Zarro et al. 1988; Canfield et al. 1990a; Falchi et al. 1992) have found red asymmetries and have interpreted this excess emission in the red wing as due to downward-moving chromospheric plasma. Canfield \& Gayley (1987) tried to match the line profiles using a model that included chromospheric condensation with moderate success. Schmieder et al. (1987) reported blue-shifted $\mathrm{H} \alpha$ absorption during the gradual phase of three major tworibbon flares, which was interpreted by the authors as due to upward chromospheric motion. Canfield et al. (1990b) found two cases of small regions with blue-shifted $\mathrm{H} \alpha$, which occurred only early in the impulsive phase. Evidences of blue asymmetries have also been reported during solar flares (Heinzel et al. 1994). Red asymmetries has often been interpreted as the result of chromospheric downward condensations (Canfield et al. 1990a). In fact, recent line profile calculations (Heinzel et al. 1994; Ding \& Fang 1997) show that the chromospheric downward condensations can explain both blue and red asymmetries.

In Fig. 5, we note that all the Balmer and Ca II lines have asymmetric profiles, at flare maximum, with red-shifted wings and blue-shifted cores. The line cores are shifted by about $-10 \mathrm{~km} \mathrm{~s}^{-1}$ from rest. The lack of blue shifted cores in the Ca II IRT $\lambda 8498$, the weakest of the Ca II IRT, could be telling us something about height discrimination. Note that the photospheric lines during the quiet state and flare state match (see Fig. 5), suggesting that these asymmetries are unlikely to be caused by a shift in the detector. Additionally, at the end of the gradual phase the profiles become symmetric as they were during the quiet state. This behaviour in the line profiles could be explained by material expanding. The blue-shift in line center could be produced by the material closest to the observer moving upwards. The red-shift in the wings would then arise from material located further down in the solar atmosphere, moving downwards. We note that the Ca II $\mathrm{H}$ and $\mathrm{K}$ lines are the only ones that show self-reversals. The central emission itself is due to the chromospheric outward temperature rise, and the central dip is a non-LTE or scattering effect due to the dilution of radiation near the surface and the corresponding decrease in excitation. The Balmer lines do not have the central emission due to their lesser dependence on temperature. Detailed analysis and modelling of the variations observed in the chromospheric line profiles during the solar flare will appear in a subsequent paper (García-Alvarez et al. 2005).

\subsection{The chromospheric and coronal flare energy budget}

In order to estimate the energy released during the solar flare, we use the line fluxes given in Table 2. We then multiply those 
Table 3. The energy encompassed by the slit and the total optical energy emitted during the solar flare at 20:07 UT on 11 January 2002.

\begin{tabular}{lcc}
\hline \hline Line & $\begin{array}{c}\text { Energy in slit } \\
\left(10^{27} \mathrm{erg}\right)\end{array}$ & $\begin{array}{c}\text { Total energy } \\
\left(10^{28} \mathrm{erg}\right)\end{array}$ \\
\hline $\mathrm{H} \alpha$ & 2.27 & 1.79 \\
$\mathrm{H} \beta$ & 1.12 & 0.88 \\
$\mathrm{H} \gamma$ & 0.84 & 0.66 \\
$\mathrm{H} \delta$ & 0.62 & 0.49 \\
$\mathrm{H} \epsilon$ & 0.53 & 0.42 \\
$\mathrm{Ca}$ II K & 1.07 & 0.84 \\
$\mathrm{Ca}$ II H & 1.52 & 1.19 \\
$\mathrm{Ca}$ II $8498 \AA$ & 1.29 & 1.04 \\
$\mathrm{Ca}$ II $8542 \AA$ & 0.95 & 0.73 \\
$\mathrm{Ca}$ II $8662 \AA$ & 0.89 & 0.70 \\
\hline Total & 11.08 & 8.72 \\
GOES $(1-8 \AA)$ & - & 3.27 \\
GOES $(0.5-4 \AA)$ & - & 0.23 \\
\hline
\end{tabular}

values by the projected slit size on the solar surface $(3.97 \times$ $10^{17} \mathrm{~cm}^{2}$ ) and integrate the value over time in order to find the energy emitted in each line. These values are shown in Table 3. This accounts for the energy encompassed by the slit during the solar flare. In order to estimate the total energy radiated during the flare, we have to multiply those values by a scaling factor. The scaling factor, equal to 7.9 , is obtained by comparing the observed area of the solar flare in the $\mathrm{H} \alpha$ images (Fig. 1) and the area of the projected slit. We have assumed that the strength of every line, during the flare, is uniform over the whole area. The total energy emitted for each line, during the flare, is then obtained by multiplying the slit energies by this scaling factor. These results are shown in Table 3 . We note that these energies are probably overestimates.

In addition, we also calculate the energy radiated in both GOES pass-bands during the flare. Integrating the radiated power between the times of our optical observations (20:07 to 20:27 UT), we obtain a total energy of $\sim 3.5 \times 10^{28} \mathrm{erg}$. Several authors obtained energy budgets for stellar flares simultaneously observed in the optical and the X-ray range (i.e., Butler et al. 1986; Doyle et al. 1988; Hawley \& Pettersen 1991). The $\mathrm{H} \gamma$ and Ca II K lines release a similar amount of energy during those stellar flares, $\sim 10^{29} \mathrm{erg}$.

The total energy emitted during the present solar flare by the $\mathrm{H} \gamma$ and $\mathrm{X}$-ray emission follows the linear logarithmic correlation found by Butler et al. (1986) for stellar flares and extended for the solar case (Butler 1993). The continuum energy release, observed in almost all stellar flares, can be even larger than the soft X-ray energy released. However, most of the flares observed on the Sun, including the one analysed in this work, do not show a detectable increase in the continuum emission, due to the relatively strong photospheric solar background.

The above values for the radiative losses are only for the upper chromosphere or the corona. (Bruner \& McWhirter 1988) have shown that, for a variety of solar plasmas, the total radiated power output, excluding hydrogen line radiation, may be estimated even if one knows the intensity of only one spectral line, e.g. O V $629 \AA$. Using the same time limits as the optical data and an area of $2.79 \times 10^{17} \mathrm{~cm}^{-2}$, we derive a total power of $1.95 \times 10^{29} \mathrm{erg}$ for the temperature interval $4.0 \leq \log T_{\mathrm{e}} \leq 8.0$. This is approximately a factor of two greater than the $\mathrm{H}$ Balmer radiative losses, and thus would suggest that adding in the additional $\mathrm{H}$ losses, e.g. the Lyman lines, that the total radiated losses in $\mathrm{H}$ would be comparable to that radiative in the other lines in the upper chromosphere, transition region and corona.

\section{Balmer decrement line modelling}

Balmer decrements (flux ratio of higher series members to $\mathrm{H} \gamma$ ) have also been used to derive plasma densities and temperatures in flare star chromospheres (Kunkel 1970; Gershberg 1974; Katsova 1990). Jevremović et al. (1998) developed a procedure (Balmer Decrement Fitting Procedure, BDFP hereafter) to fit the Balmer decrement in order to determine various physical parameters in the flaring plasma. García-Alvarez et al. (2002), based on the BDFP, obtained the first detailed trace of physical parameters during a stellar flare. These authors also applied the BDFP to literature data from a solar flare (Johns-Krull et al. 1997) and from a a stellar flare (Katsova 1990) obtaining values for the physical parameters in good agreement with those reported in those respective works.

The BDFP is based on the solution of the radiative transfer equation, using the escape probability technique (Drake 1980; Drake \& Ulrich 1980) and a simplified picture of the flaring plasma as a slab of hydrogen with an underlying thermal source of radiation which causes photoionization. This source, which represents the photosphere, can be exposed to additional heating during the flare. This procedure assumes that the Balmer lines are formed in the upper chromosphere. We note that this simplified picture leaves out some important processes (i.e. cooling from $\mathrm{Mg}$ II, C II and other metals). The BDFP minimizes the difference between the observed and calculated Balmer decrement using a multi-directional search algorithm (Torczon 1991, 1992). This allows us to find the best possible solution for the Balmer decrement in a four dimensional parameter space where the parameters are: electron temperature, electron density, optical depth in the Ly $\alpha$ line, and the temperature of the underlying source (background temperature). The best solutions for the Balmer decrement allow us to calculate the effective thickness of the slab of hydrogen plasma with which we are able to determine the surface area of the emitting plasma from the calculated emission measure. Although the temperature of the underlying source could be a free parameter in our code, we have fixed a lower limit at $5500 \mathrm{~K}$ for numerical stability reasons.

We apply the BDFP to the C 7.5 solar flare observed during our solar campaign. We normalised the flare-only fluxes for $\mathrm{H} \alpha, \mathrm{H} \beta, \mathrm{H} \gamma, \mathrm{H} \delta$ and $\mathrm{H} \epsilon$ to $\mathrm{H} \gamma$ for three intervals around flare maximum.

In Fig. 7 we plot the observed and the fitted Balmer decrements for the solar flare. We note from the plots that, the slope of the Balmer decrement is shallower for the flare maximum 

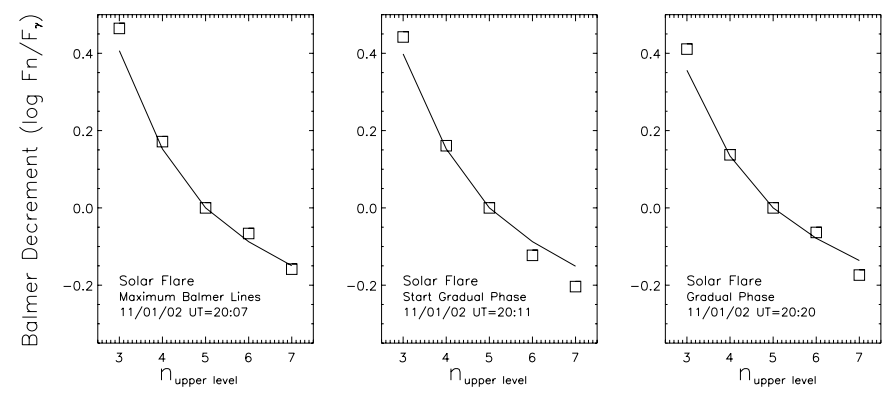

Fig. 7. The observed Balmer decrements (squares) and the optimum computed fits (solid line) at different times during the maximum and gradual phases of the solar flare observed during the solar campaign on the CAT, Lick Observatory.

than for the other two, as seen in stellar flares. We applied the BDFP to the Balmer decrements observed around flare maximum. The electron temperatures we obtain are around $8000 \mathrm{~K}$ for the first five Balmer decrements observed during the flare. The electron densities range from $5 \times 10^{13}$ to $7 \times 10^{13} \mathrm{~cm}^{-3}$, roughly the same as García-Alvarez et al. (2002) obtained for the solar flare observed by Johns-Krull et al. (1997). The electron densities found for the solar flare imply that the Balmer emitting plasma originates somewhere in the chromosphere, where the electron density range from $\sim 10^{15} \mathrm{~cm}^{-3}$ to $\sim 10^{12} \mathrm{~cm}^{-3}$ (Gabriel \& Mason 1982). The effective thickness of the slab of hydrogen plasma around flare maximum is $\sim 100 \mathrm{~km}$. The value for $\log \tau(\operatorname{Ly} \alpha)$ are around 4.5 , which means that the plasma is optically thick in this line.

We have used the best fit solutions for the Balmer decrement of the solar flare combined with the derived flux for the $\mathrm{H} \beta$ line, to calculate the surface area of the emitting plasma. The value we obtain is $\sim 4.9 \times 10^{18} \mathrm{~cm}^{2}$. This value is roughly the same as that inferred from the BBSO image obtained during flare maximum $\left(3 \times 10^{18} \mathrm{~cm}^{-2}\right)$. Comparing the values for the physical parameters obtained here (C7.5 flare) with those obtained by García-Alvarez et al. (2002) for the M 7.7 flare observed by Johns-Krull et al. (1997), we find slightly larger values for all the model parameters in the stronger flares.

\section{Summary}

We have presented a multi-wavelength analysis of a solar flare observed at 20:07 UT on 11 January 2002 which took place in the active region NOAA 9773. The main results of this work are:

1. The flare shows several eruptive centers in both the $\mathrm{H} \alpha$ and $\mathrm{Ca}$ II $\mathrm{K}$ images. The $\mathrm{H} \alpha$ and $\mathrm{Ca}$ II $\mathrm{K}$ images consist of two components, a bright kernel during the impulsive phase and bright loops during the gradual phase.

2. The main chromospheric activity indicators show either filling-in (Balmer lines) or emission (Ca II lines) during the flare. Photospheric lines such as the MgI triplet and Fe I lines also show filling-in during the event. This may indicate that the flare reaches the lower chromosphere. The $\mathrm{He} \mathrm{I}_{3}$ line appears in absorption during the flare, being undetectable during the quiet state.
3. We observe that all the Balmer and Ca II lines show asymmetric profiles, with red-shifted wings and blue-shifted cores. This behaviour could be explained by material expanding. The blue-shift in the line center could be produced by the material closest to the observer moving upwards. The red-shift in the wings would then arise from material located further down in the solar atmosphere, moving downwards.

4. We observe that the Ca II lines decline more slowly than the Balmer lines, as is observed in $\mathrm{M}$ dwarf flares. The photospheric lines (Mg I Triplet) and metallic lines (Fe I) have their maximum enhancement roughly at the same time as the chromospheric lines, which suggests that the energy supply to the photosphere would be the same. The temporal behaviour suggests that all the chromospheric lines were formed in the same atmospheric region.

5. In the EUV lines, observing a close but different kernel to the Lick pointing, we detect a precursor brightening in the transion region $\mathrm{OV}$ line peaking $10 \mathrm{~min}$ before the flare maximum. After the flare, the evolution shows a typical pattern of hot loops cooling down from $8 \times 10^{6} \mathrm{~K}$ (Fe XIX line) to transition region $(250000 \mathrm{~K})$ temperatures in an interval of around $20 \mathrm{~min}$.

6. Although the estimates for the energy released in the optical lines and X-rays are crude, a total flare energy in excess of $10^{29}$ erg was derived. The optical flare showed no detectable increase in the continuum emission, as happens in most of the flares observed on the Sun, probably due to the relatively strong photospheric solar background.

7. We have determined the main chromospheric physical parameters for the flare maximum. We found electron densities of $\sim 10^{14} \mathrm{~cm}^{-3}$ and electron temperatures of $\sim 8000 \mathrm{~K}$ around flare maximum implying that the Balmer emitting plasma originates somewhere in the chromosphere, rather than in the photosphere. The modelling method also yields an estimate of the flare emitting area $(4.9 \times$ $10^{18} \mathrm{~cm}^{-2}$ ) which is roughly the same that the observed value $\left(3 \times 10^{18} \mathrm{~cm}^{-2}\right)$.

There is an obvious need for more solar flare observations of this kind to be compared with stellar flares, particularly if one can obtain simultaneous coverage with UV and X-ray satellites. Furthermore, on the Sun, due to its proximity, we can study flares in a very detailed way in all the wavelength ranges (optical, X-ray, etc.). So, observing the Sun in a similar way to which we observe the stars will, hopefully, give a better understanding of the unresolved processes causing the observed stellar activity.

Acknowledgements. Research at Armagh Observatory is grant-aided by the Department of Culture, Arts and Leisure for N. Ireland. We thank the BBSO for the provision of telescope time and to the observing staff for their support in obtaining the data. C.M.J.-K. acknowledges support through NSF grant ATM-9809970. We would like to thank the CDS and TRACE teams for their help. This work was supported by part by PPARC Visitors grant PPA/V/S/2003/00044 and by a PRTLI research grant for Grid-enabled Computational Physics of Natural Phenomena (Cosmogrid). SOHO is a project of international cooperation between ESA and NASA. 


\section{References}

Acampa, E., Smaldone, L. A., Sambuco, A. M., \& Falciani, R. 1982, A\&AS, 47, 485

Allen, C. W. 1976, Astrophysical Quantities (Astrophysical Quantities, London: Athlone), 3rd edition

Andretta, V., \& Giampapa, M. S. 1995, ApJ, 439, 405

Basri, G., Wilcots, E., \& Stout, N. 1989, PASP, 101, 528

Bocchialini, K., \& Gouttebroze, P. 1996, A\&A, 313, 949

Brosius, J. W. 2003, ApJ, 586, 1417

Brosius, J. W., \& Phillips, K. J. H. 2004, ApJ, 613, 580

Bruner, M. E., \& McWhirter, R. W. P. 1988, ApJ, 326, 1002

Butler, C. J. 1993, A\&A, 272, 507

Butler, C. J., Rodono, M., Foing, B. H., \& Haisch, B. M. 1986, Nature, 321,679

Canfield, R. C., \& Gayley, K. G. 1987, ApJ, 322, 999

Canfield, R. C., Metcalf, T. R., Zarro, D. M., \& Lemen, J. R. 1990a, ApJ, 348, 333

Canfield, R. C., Penn, M. J., Wulser, J., \& Kiplinger, A. L. 1990b, ApJ, 363, 318

Cheng, C.-C., Tandberg-Hanssen, E., Bruner, E. C., et al. 1981, ApJ, 248, L39

Dempsey, R. C., Linsky, J. L., Fleming, T. A., \& Schmitt, J. H. M. M. 1993, ApJS, 86, 599

Dennis, B. R. 1985, Sol. Phys., 100, 465

Dennis, B. R., \& Schwartz, R. A. 1989, Sol. Phys., 121, 75

Ding, M. D., \& Fang, C. 1997, A\&A, 318, L17

Donati-Falchi, A., Smaldone, L. A., \& Falciani, R. 1984, A\&A, 131, 256

Doyle, J. G., Butler, C. J., Byrne, P. B., \& van den Oord, G. H. J. 1988, A\&A, 193, 229

Drake, S. A. 1980, Ph.D. Thesis, 72

Drake, S. A., \& Ulrich, R. K. 1980, ApJS, 42, 351

Falchi, A., Falciani, R., \& Smaldone, L. A. 1992, A\&A, 256, 255

Foing, B. H., Crivellari, L., Vladilo, G., Rebolo, R., \& Beckman, J. E. 1989, A\&AS, 80, 189

Foukal, P. 1990, Solar astrophysics (New York: Wiley-Interscience), 492

Gabriel, A. H., \& Mason, H. E. 1982, in Applied atomic collision physics, Volume 1 (A84-15476 04-46) (New York and London, Academic Press), 1, 345

Garcia Alvarez, D. 2000, Irish Astron. J., 27, 117

García-Alvarez, D., Jevremović, D., Doyle, J. G., \& Butler, C. J. 2002, A\&A, 383, 548

García-Alvarez, D., Foing, B. H., Montes, D., et al. 2003, A\&A, 397, 285

García-Alvarez, D., Avrett, E. H., Doyle, J. G., \& Johns-Krull, C. M. 2005, A\&A, in preparation

Gershberg, R. E. 1974, AZh, 51, 552

Green, L. M., Démoulin, P., Mandrini, C. H., \& Van Driel-Gesztelyi, L. 2003, Sol. Phys., 215, 307

Gunn, A. G., \& Doyle, J. G. 1997, A\&A, 318, 60

Haisch, B., Strong, K. T., \& Rodono, M. 1991, ARA\&A, 29, 275

Hale, G. E., Ellerman, F., Nicholson, S. B., \& Joy, A. H. 1919, ApJ, 49, 153

Handy, B. N., Acton, L. W., Kankelborg, C. C., et al. 1999, Sol. Phys., 187,229
Harra, L. K., Matthews, S. A., \& Culhane, J. L. 2001, ApJ, 549, L245

Harrison, R. A., McNamara, B. J., Pedersen, H., et al. 1995, Sol. Phys., 162,233

Hawley, S. L., \& Pettersen, B. R. 1991, ApJ, 378, 725

Heinzel, P., Karlicky, M., Kotrc, P., \& Svestka, Z. 1994, Sol. Phys., 152,393

Houdebine, E. R. 2003, A\&A, 397, 1019

Houdebine, E. R., Butler, C. J., Panagi, P. M., Rodono, M., \& Foing, B. H. 1991, A\&AS, 87, 33

Huenemoerder, D. P. 1986, AJ, 92, 673

Jevremović, D., Butler, C. J., Drake, S. A., O’Donoghue, D., \& van Wyk, F. 1998, A\&A, 338, 1057

Johns-Krull, C. M., Hawley, S. L., Basri, G., \& Valenti, J. A. 1997, ApJS, 112, 221

Katsova, M. M. 1990, SvA, 34, 614

Kunkel, W. E. 1970, ApJ, 161, 503

Landman, D. A. 1981, ApJ, 244, 345

Lin, R. P. 1974, in Coronal Disturbances, 201

Linsky, J. L., Hunten, D. M., Sowell, R., Glackin, D. L., \& Kelch, W. L. 1979, ApJS, 41, 481

Mauas, P. J. D. 1990, ApJS, 74, 609

Mauas, P. J. D., Machado, M. E., \& Avrett, E. H. 1990, ApJ, 360, 715

Mirzoyan, L. V. 1984, Vistas Astron., 27, 77

Montes, D., Fernandez-Figueroa, M. J., de Castro, E., \& Sanz-Forcada, J. 1997, A\&AS, 125, 263

Montes, D., \& Ramsey, L. W. 1998, A\&A, 340, L5

Montes, D., Saar, S. H., Collier Cameron, A., \& Unruh, Y. C. 1999, MNRAS, 305, 45

Neidig, D. F. 1989, Sol. Phys., 121, 261

Oliveira, J. M., \& Foing, B. H. 1999, A\&A, 343, 213

Pettersen, B. R. 1989, Sol. Phys., 121, 299

Pierce, A. K., \& Waddell, J. H. 1961, MmRAS, 63, 89

Scherrer, P. H., et al. 1995, Sol. Phys., 162, 129

Schmieder, B., Forbes, T. G., Malherbe, J. M., \& Machado, M. E. 1987, ApJ, 317, 956

Shine, R. A., \& Linsky, J. L. 1972, Sol. Phys., 25, 357

Shine, R. A., \& Linsky, J. L. 1974, Sol. Phys., 39, 49

Svestka, Z. 1976 (Dordrecht, D. Reidel Publishing Co.), Geophysics and Astrophysics Monographs, 415, 8

Tandberg-Hanssen, E. 1967, Solar activity (Waltham: Mass.: Blaisdell)

Tandberg-Hanssen, E., \& Emslie, A. G. 1988, The physics of solar flares (Cambridge and New York: Cambridge University Press), 1988, 286

Torczon, V. 1991, SIAM J. Optimization, 1, 123

Torczon, V. 1992, Tech. Report 92-9, Dep. of Mathematical Sciences, Rice University, Houston

Valenti, J. A. 1994, Ph.D. Thesis, 187

Vernazza, J. E., Avrett, E. H., \& Loeser, R. 1981, ApJS, 45, 635

Vogt, S. S. 1987, PASP, 99, 1214

Woodgate, B. E., Shine, R. A., Poland, A. I., \& Orwig, L. E. 1983, ApJ, 265, 530

Zarro, D. M., Canfield, R. C., Metcalf, T. R., \& Strong, K. T. 1988, ApJ, 324, 582

Zirin, H. 1975, ApJ, 199, L63

Zirin, H. 1988, Annual Scientific Supplement to Urania

Zirin, H., Liggett, M., \& Patterson, A. 1982, Sol. Phys., 76, 387 